\title{
Implementing Grover's Quantum Search on a Para-hydrogen based Pure State NMR Quantum Computer
}

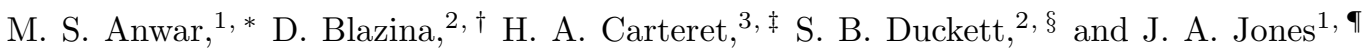 \\ ${ }^{1}$ Centre for Quantum Computation, Clarendon Laboratory, \\ University of Oxford, Parks Road, OX1 3PU, United Kingdom \\ ${ }^{2}$ Department of Chemistry, University of York, Heslington, York, YO10 5DD, United Kingdom \\ ${ }^{3}$ LITQ, Departement d'Informatique et Recherche Opérationelle, \\ Pavillon André-Aisenstadt, Université de Montréal, Montréal, Québec H3C 3J7, Canada
}

(Dated: July 7, 2018)

\begin{abstract}
We demonstrate the implementation of Grover's quantum search algorithm on a liquid state nuclear magnetic resonance (NMR) quantum computer using essentially pure states. This was achieved using a two qubit device where the initial state is an essentially pure $(\varepsilon=1.06 \pm 0.04)$ singlet nuclear spin state of a pair of ${ }^{1} \mathrm{H}$ nuclei arising from a chemical reaction involving para-hydrogen. We have implemented Grover's search to find one of four inputs which satisfies a function.
\end{abstract}

PACS numbers: 03.67.Lx, 82.56.-b, 03.67.Mn

\section{INTRODUCTION}

Quantum computers [1] are of enormous interest because of their ability to perform calculations which appear to be intractable on any conceivable classical computer. The leading technology for the implementation of quantum logic gates is liquid state nuclear magnetic resonance (NMR) 2, 3, 4], and NMR quantum computers [5, 6, 7, 8] have been used to implement a range of quantum algorithms, mostly notably Shor's algorithm which was used to factor fifteen [9]. Unfortunately NMR devices have one great disadvantage: the small energy gap between the Zeeman levels that are used as computational basis states means that direct cooling is not a practical method for obtaining a pure initial state. The most common way round this is to prepare a pseudopure state [5], whose behaviour is identical to that of a pure state up to a scaling factor, but this is not a good solution for two reasons. Firstly, the efficiency of pseudopure state preparation falls off exponentially with the number of qubits in the quantum computer [10], which means that NMR devices cannot be scaled up to useful sizes, and secondly the states used are provably separable 11], and so cannot exhibit the phenomenon of entanglement.

These problems are not inherent in NMR quantum computation, but arise from the use of pseudopure states prepared from high temperature thermal states. We have recently shown how high purity initial states can be prepared using para-hydrogen techniques [12, 13], and that these states can be used to perform the simplest quantum computation, Deutsch's algorithm [14]. Similar results have been obtained by Hübler et al. 15], albeit

\footnotetext{
*Electronic address: muhammad.anwar@physics.ox.ac.uk

${ }^{\dagger}$ Electronic address: db30@york.ac.uk

‡Electronic address: cartereh@iro.umontreal.ca

$\S$ Electronic address: sbd3@york.ac.uk

๑Electronic address: jonathan.jones@qubit.org
}

with a lower spin state purity. Here we describe the use of a new molecular system which allows essentially pure initial states to be generated, and the implementation of Grover's quantum search algorithm 16] on our pure state computer. State initialization on demand is achieved by using a 12 ns laser pulse to initiate a rapid chemical reaction, and is therefore both well controlled and time coherent with the NMR radiofrequency pulses.

\section{PARA-HYDROGEN}

We prepare pure spin states using an effect called parahydrogen induced polarization (PHIP) [17, 18, 19, 20]. The existence of the para isomer of dihydrogen, $\mathrm{H}_{2}$, is a consequence of the Pauli principle, which requires the overall wavefunction to be antisymmetric with respect to exchange of the fermionic ${ }^{1} \mathrm{H}$ nuclei. It follows that $\mathrm{H}_{2}$ molecules in even rotational states, most notably the $\mathrm{J}=0$ ground state, possess an antisymmetric nuclear spin wave function and correspond to nuclear spin singlets, termed para. Thus if $\mathrm{H}_{2}$ is cooled to a temperature of $20 \mathrm{~K}$ in the presence of a paramagnetic catalyst then essentially pure para-hydrogen will be obtained, with the singlet spin wavefunction

$$
\left|\psi^{-}\right\rangle=\frac{1}{\sqrt{2}}(|01\rangle-|10\rangle),
$$

where we have used the computational basis in which $|0\rangle$ corresponds to the ground state and $|1\rangle$ to the excited state of a spin- $1 / 2$ particle. The para-hydrogen molecule cannot be used directly for NMR quantum computing, due to its high symmetry, but this can be overcome by using a chemical reaction to prepare a new molecule, in which the two hydrogen atoms can be made distinct and can be separately addressed. For further details see 12, 13, 14, 15, 17, 18, 19, 20].

In our previous work we have used the two hydride ${ }^{1} \mathrm{H}$ nuclei in $\mathrm{Ru}(\mathrm{H})_{2}(\mathrm{CO})_{2}($ dppe $)$, where dppe indicates 


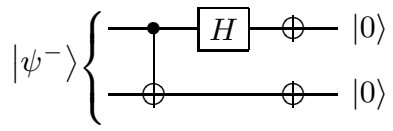

FIG. 1: Quantum circuit to convert a singlet state $\left|\psi^{-}\right\rangle$into the conventional initial state $|00\rangle . H$ indicates a single qubit Hadamard gate, $\oplus$ indicates a single qubit NOT gate, and the initial two qubit gate is a controlled-NOT gate.

1,2-bis(diphenylphosphino)ethane, as our NMR quantum computer. Detailed analysis of this system shows that the spins are not prepared in an absolutely pure singlet state, but in a slightly mixed state, which can be easily converted to a Werner state [21] of the form

$$
\rho_{\text {init }}=(1-\varepsilon) \frac{\mathbf{1}}{4}+\varepsilon\left|\psi^{-}\right\rangle\left\langle\psi^{-}\right|,
$$

with a spin-state purity of $\varepsilon=0.898 \pm 0.026[13$. Although this system is adequate for an initial proof of principle experiment, it is not ideally suited for quantum computing; in particular its ${ }^{1} \mathrm{H}$ spin-spin relaxation time $\left(\mathrm{T}_{2}=0.58 \mathrm{~s}\right)$ is uncomfortably short. We have, therefore, prepared a novel but closely related system, $\mathrm{Ru}(\mathrm{H})_{2}(\mathrm{CO})_{2}$ (dpae), where dpae indicates 1,2bis(diphenylarsino)ethane. This system has a slightly longer ${ }^{1} \mathrm{H}$ spin-spin relaxation time $\left(\mathrm{T}_{2}=0.67 \mathrm{~s}\right)$, and has the further advantage that the initial state can be prepared with a purity indistinguishable from one (the purity was measured using techniques described previously [12, 13] and was found to be $\varepsilon=1.06 \pm 0.04$ ). Thus we describe our new system as a pure state NMR quantum computer.

Although our quantum computer starts in a pure state, it does not start in the state conventionally assumed for two qubit quantum computers, that is $|00\rangle$ in the computational basis. It is, however, simple to convert our initial state to this form by using a disentangling circuit, such as that shown in Fig. [1]

\section{GROVER'S QUANTUM SEARCH}

Grover's quantum search algorithm 16 permits an efficient search for one of $k$ matching items within a search space of size $n$. The algorithm is most conveniently described in terms of a binary function, with $n$ possible inputs of which $k$ satisfy the function, that is produce an output of 1 . The function is assumed to be implemented by means of an oracle, which will produce values of the function for any given input, but does not permit any other method of analyzing the function. The best classical algorithm is simply to try inputs at random, and this will allow a satisfying input to be located in $O(n / k)$ queries. By contrast, Grover's quantum search allows a satisfying input to be located in $O(\sqrt{n / k})$ queries. The simplest case occurs when $k=1$ and $n=4$, in which

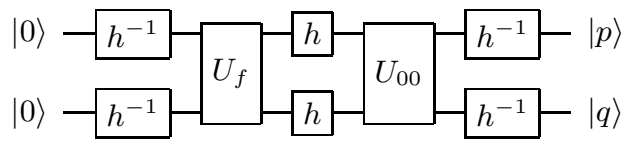

FIG. 2: A quantum circuit to implement Grover's search algorithm on a two qubit quantum computer. Following previous practice 22] Hadamard gates $H$ have been replaced by pseudo-Hadamard gates $h$ and $h^{-1}$, which correspond to $90_{ \pm y}^{\circ}$ rotations. The function being studied is encoded in the propagator $U_{f}$, and $U_{00}$ replaces $|00\rangle$ by $-|00\rangle$ while leaving other basis states unchanged. At the end of the computation the two qubits are left in states corresponding to the satisfying input, that is $f(p q)=1$.

case the satisfying input can be located in a single query, and this is the case we will concentrate on here.

The key element in an implementation of Grover's search is a quantum function evaluation oracle, $U_{f}$, which performs the transformation 22]

$$
|x\rangle \stackrel{U_{f}}{\longrightarrow}(-1)^{f(x)}|x\rangle
$$

where $|x\rangle$ indicates a quantum register in a state corresponding to the input $x$, and the function $f$ has values of 0 or 1 . Typically $x$ is written in binary form, and $|x\rangle$ is a set of qubits with values corresponding to successive bits of $x$. A quantum circuit which can be used to locate one satisfying input from four using this oracle is shown in Fig. 2

\section{THE EXPERIMENT}

Our two qubit system comprises the hydride ${ }^{1} \mathrm{H}$ nuclei in $\mathrm{Ru}(\mathrm{H})_{2}(\mathrm{CO})_{2}$ (dpae), where the hydride atoms are derived from para-hydrogen. The precursor compound $\mathrm{Ru}(\mathrm{CO})_{3}$ (dpae) was prepared from $\mathrm{Ru}_{3}(\mathrm{CO})_{12}$ and dpae using techniques described previously 12, 23]. Essentially pure para-hydrogen was prepared at a temperature of $18 \mathrm{~K}$ using a charcoal-based catalyst, and was introduced into a $5 \mathrm{~mm} \mathrm{NMR}$ tube containing $\mathrm{Ru}(\mathrm{CO})_{3}$ (dpae) dissolved in $\mathrm{d}_{6}$-benzene. The NMR tube was then transferred into a $400 \mathrm{MHz}$ spectrometer fitted with a ${ }^{1} \mathrm{H} /{ }^{31} \mathrm{P}$ probe modified for in situ photolysis 24]. The spectrometer triggered a $12 \mathrm{~ns}$ UV pulse of wavelength $308 \mathrm{~nm}$ from an MPB Technologies MSX-250 pulsed XeCl excimer laser, irradiating the active region of the NMR sample and producing the unstable species $\mathrm{Ru}(\mathrm{CO})_{2}$ (dpae). Intermediates of this type have been shown to react with hydrogen on the sub-microsecond timescale 25], which would in this case lead to the formation of $\mathrm{Ru}(\mathrm{H})_{2}(\mathrm{CO})_{2}$ (dpae). For further details see 12,13 , 14].

The hydride resonances appear at $-7.61 \mathrm{ppm}$ and $-7.21 \mathrm{ppm}$, with a frequency separation of $\delta=160 \mathrm{~Hz}$, while the hydride $J$ coupling $\left({ }^{2} J_{\mathrm{HH}}\right)$ was $4.8 \mathrm{~Hz}$. The ${ }^{1} \mathrm{H}$ transmitter frequency was placed exactly between the two resonance frequencies. The laser flash acts as an 
initialisation switch, generating the pure state $\left|\psi^{-}\right\rangle$on demand, which is subsequently used for the implementation.

The singlet state $\left|\psi^{-}\right\rangle$can be converted to the desired initial state $|00\rangle$ using the NMR pulse sequence

$$
P_{\text {prep }} \equiv\left[\frac{1}{4 \delta}\right] 90_{y}\left[\frac{1}{4 J}\right] 180_{x}\left[\frac{1}{4 J}\right] 180_{y}\left[\frac{1}{2 \delta}\right] 90_{x}
$$

which has been described previously 14]. This pulse sequence is composed of a series of hard RF pulses (which excite both spins equally) and periods of evolution under the background Hamiltonian, indicated by enclosing the appropriate evolution time in brackets. Following NMR conventions operations are applied from left to right. No selective pulses are used, but at two points equal and opposite $z$ rotations are applied to the two qubits. These were achieved by evolution under the Zeeman Hamiltonian at frequencies of $\pm \delta / 2 \mathrm{~Hz}$, with evolution under the small $J$ coupling neglected over these short periods. NMR pulse sequences to implement the four possible functions $f$ with a single satisfying value can be developed in the same way and are shown below, where each function is identified by listing its satisfying input.

$$
\begin{aligned}
P_{00} & \equiv\left[\frac{1}{4 J}\right] 90_{-x} 90_{y} 90_{-x}\left[\frac{1}{4 J}\right] 180_{x} \\
P_{01} & \equiv\left[\frac{1}{4 J}\right] 180_{x}\left[\frac{1}{4 J}\right]\left[\frac{1}{2 \delta}\right] 180_{x} \\
P_{10} & \equiv\left[\frac{1}{2 \delta}\right]\left[\frac{1}{4 J}\right] 180_{x}\left[\frac{1}{4 J}\right] 180_{x} \\
P_{11} & \equiv\left[\frac{1}{4 J}\right] 180_{x}\left[\frac{1}{4 J}\right] 90_{-x} 90_{y} 90_{-x}
\end{aligned}
$$

Two of these functions require equal $z$ rotations to be applied to the two qubits, and these were achieved using composite $z$ pulses.

The final element used in our pulse sequences is a gradient pulse, indicated by crush. This is a short period of evolution during which time the magnetic field is temporarily made spatially inhomogeneous, so that spins in different parts of the sample experience different Larmor frequencies. These crush pulses were applied immediately before and after the Grover circuit, at which points the qubits should be in eigenstates of the computational basis. These states do not evolve under magnetic fields, and should not be affected by the crush pulse, but most error terms which can arise will be dephased 22.

Putting these elements together gives the final pulse sequence used to implement Grover's search,

$$
P_{\text {prep }} \text { crush } 90_{-y} P_{f} 90_{y} P_{00} 90_{-y} \text { crush } 90_{y} \text { acquire }
$$

where $P_{f}$ corresponds to one of the four pulse sequences shown in equation 5 . The state of the spin system is analyzed by applying a hard $90^{\circ}$ pulse and observing the resulting NMR spectrum. With appropriate phasing the state of each qubit is then indicated by multiplets point-
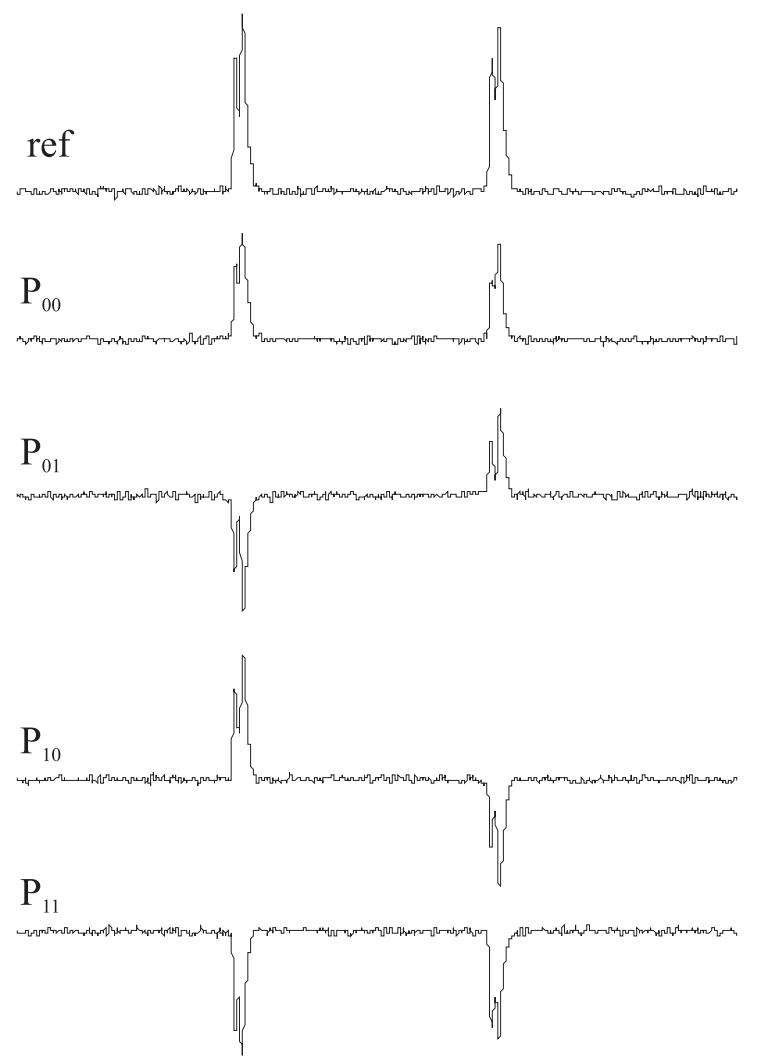

FIG. 3: Experimental spectra from our implementation of Grover's quantum search. The top spectrum is a phase reference acquired from our computer in the state $|00\rangle$. The four lower spectra were acquired after implementing Grover's search with the oracle set to each of the four functions in turn.

ing upwards for $|0\rangle$ or downwards for $|1\rangle$. A phase reference spectrum is easily obtained using the sequence

$$
P_{\text {prep }} \text { crush } 90_{y} \text { acquire }
$$

and the experimental results are shown in Fig. 3.

These spectra show exactly the pattern expected: each computation ends with the system in one of the four basis states, and the final state corresponds with the satisfying input of the corresponding function. Note that in this paper we have used the same conventions as in [14], where the first qubit occurs on the right hand side of the NMR spectrum; this is the reverse of the convention used in 22. There are small imperfections visible in the spectra: in particular there are imbalances in the intensities of the two multiplets and of the two lines in each multiplet, and the overall signal intensity is lower in spectra obtained from quantum computations than in the phase reference spectrum. The imbalances can be ascribed to errors in the implementations of the logic gates, while the signal loss is largely a consequence of relaxation during the pulse sequences. The effects of relaxation are less marked in this study than in our previous implementation of Deutsch's algorithm 14]; this partly reflects the longer relaxation times in our new spin system, but is 
also a consequence of the symmetry in the gates applied to each qubit in Grover's algorithm.

These results confirm that it is possible to use parahydrogen techniques to implement quantum algorithms on a pure state NMR quantum computer. We are now seeking to extend these results to larger spin systems and more complex algorithms.

\section{Acknowledgements}

We thank the EPSRC for financial support. MSA thanks the Rhodes Trust for a Rhodes Scholarship. HAC thanks MITACS for financial support. Quantum circuits were drawn using Q-circuit [26].
[1] C. H. Bennett, D. P. DiVincenzo, Nature 404 (2000) 247.

[2] R. R. Ernst, G. Bodenhausen, and A. Wokuan, Principles of Nuclear Magnetic Resonance in One and Two Dimensions (Clarendon Press, Oxford, 1987).

[3] M. H. Levitt, Spin Dynamics: Basics of Nuclear Magnetic Resonance (John Wiley \& Sons, 2001).

[4] R. Freeman, Spin Choreography: Basic Steps in High Resolution NMR (Oxford University Press, Oxford, 1998).

[5] D. G. Cory, A. F. Fahmy, T. F. Havel, in "PhysComp '96' (T. Toffoli, M. Biafore and J. Leão, Eds.), pp. 8791, New England Complex Systems Institute (1996)

[6] D. G. Cory, A. F. Fahmy, T. F. Havel, Proc. Natl. Acad. Sci. USA 94 (1997) 1634.

[7] J. A. Jones, M. Mosca, J. Chem. Phys. 109 (1998) 1648.

[8] I. L. Chuang, N. Gershenfeld, M. Kubinec, Phys. Rev. Lett 80 (1998) 3408.

[9] L. M. K. Vandersypen, M. Steffen, G. Breyta, C. S. Yannoni, M. H. Sherwood, I. L. Chuang, Nature 414 (2001) 883.

[10] W. S. Warren, Science 277 (1997) 229.

[11] S. L. Braunstein, C. M. Caves, R. Jozsa, N. Linden, S. Popescu, R. Schack, Phys. Rev. Lett. 83 (1999) 1054.

[12] M. S. Anwar, D. Blazina, H. A. Carteret, S. B. Duckett, T. K. Halstead, J. A. Jones, C. M. Kozak, R. J. K. Taylor, Phys. Rev. Lett. (2004, in press); e-print quant-ph/0312014

[13] D. Blazina, S. B. Duckett, T. K. Halstead, R. J. K. Taylor, M. S. Anwar, J. A. Jones, H. A. Carteret, submitted to J. Phys. Chem. A (2004).

[14] M. S. Anwar, J. A. Jones, D. Blazina, S. B. Duckett, H. A. Carteret, submitted to Phys. Rev. A (2004); eprint quant-ph/0406044

[15] P. Hübler, J. Bargon, and S. J. Glaser, J. Chem. Phys. 113, 2056 (2000).

[16] L. K. Grover, Phys. Rev. Lett. 79 (1997) 325.

[17] C. R. Bowers, D. P. Weitekamp, Phys. Rev. Lett. 57 (1986) 2645.

[18] J. Natterer, J. Bargon, Prog. Nucl. Magn. Reson. Spectros. 31 (1997) 293.

[19] S. B. Duckett, C. J. Sleigh, Prog. Nucl. Magn. Reson. Spectros. 34 (1999) 71.

[20] S. B. Duckett, D. Blazina, Eur. J. Inorg. Chem. 16 (2003) 2901.

[21] R. F. Werner, Phys. Rev. A 40 (1989) 4277.

[22] J. A. Jones, M. Mosca, and R. H. Hansen, Nature 393 (1998) 344.

[23] D. Schott, C. J. Sleigh, J. P. Lowe, S. B. Duckett, R. J. Mawby, and M. G. Partridge, Inorg. Chem, 41, 2960, (2002).

[24] C. Godard, P. Callaghan, J. L. Cullingham, S. B. Duckett, J. A. B. Lohman, and R. N. Perutz, Chem. Comm. 23 (2003) 2386.

[25] L. Cronin, M. C. Nicasio, R. N. Perutz, R. G. Peters, D. M. Roddick, and M. K. Whittlesey, J. Am. Chem. Soc. 117 (1995) 10047.

[26] B. Eastin and S. T. Flammia, e-print quant-ph/0406003 